\title{
FYN promotes breast cancer progression through epithelial-mesenchymal transition
}

\author{
YE-GONG XIE ${ }^{1-4^{*}}, \mathrm{YUE} \mathrm{YU}^{1-3^{*}}, \mathrm{LI}^{-K U N} \mathrm{HOU}^{1-3}, \mathrm{XIN}^{\mathrm{WANG}}{ }^{1-3}, \mathrm{BIN} \mathrm{ZHANG}^{1-3}$ and XU-CHEN CAO ${ }^{1-3}$ \\ ${ }^{1}$ The First Department of Breast Cancer, Tianjin Medical University Cancer Institute and Hospital, \\ National Clinical Research Center for Cancer, Tianjin 300060; ${ }^{2}$ Key Laboratory of Cancer Prevention and Therapy, \\ Tianjin Medical University, Tianjin 300060; ${ }^{3}$ Key Laboratory of Breast Cancer Prevention and Therapy, \\ Tianjin Medical University, Ministry of Education, Tianjin 300060, ${ }^{4}$ Department of Anesthesiology, \\ Tianjin Medical University General Hospital, Tianjin 300052, P.R. China
}

Received February 2, 2016; Accepted March 10, 2016

DOI: 10.3892/or.2016.4894

\begin{abstract}
FYN, one of the members of the Src family of kinases (SFKs), has been reported to be overexpressed in various types of cancers and correlated with cell motility and proliferation. However, the mechanism is still unclear. In the present study, we found that FYN was overexpressed in breast cancer and overexpression of FYN promoted cell proliferation, migration and invasion in the MCF10A cells, whereas depletion of FYN suppressed cell proliferation, migration and invasion in the MDA-MB-231 cells. Moreover, FYN upregulated the expression of mesenchymal markers and epithelial-mesenchymal transition (EMT)-related transcription factors, and downregulated the expression of epithelial markers, suggesting that FYN induces EMT in breast cancer cells. Furthermore, FYN was transcriptionally regulated by FOXO1 and mediated FGF2-induced EMT through both the PI3K/AKT and ERK/MAPK pathways.
\end{abstract}

\section{Introduction}

Breast cancer is the most common type of malignant tumor and the most deadly cancer among women worldwide (1). In the Asia-Pacific region, the incidence of breast cancer has increased in recent years and is rising year by year (2). Although sophisticated methods including surgery, chemotherapy, radiotherapy and biotherapy are being used in breast cancer treatment, the

Correspondence to: Professor Xu-Chen Cao or $\mathrm{Dr} B$ Bin Zhang, The First Department of Breast Cancer, Tianjin Medical University Cancer Institute and Hospital, Huanhuxi Road, Hexi, Tianjin 300060, P.R. China

E-mail: caoxuchen@tmu.edu.cn

E-mail: eeflying@163.com

${ }^{*}$ Contributed equally

Key words: FYN, fibroblast growth factor 2, epidermal growth factor, epithelial-mesenchymal transition, breast cancer, progression mortality rate of breast cancer patients is still high and this trend is anticipated to continue (3). Among numerous causes, metastasis is the major cause of cancer-related mortality in breast cancer patients (4). Thus, the molecular mechanism of metastasis is a key focus of breast cancer research.

Epithelial-mesenchymal transition (EMT) which plays an important role in cancer metastasis, is an embryonic transdifferentiation process converting adjacent epithelial cells with polarity to mesenchymal cells. $(5,6)$. During the EMT process, cells lose epithelial markers, such as E-cadherin and $\beta$-catenin, and gain increased levels of mesenchymal markers, such as $\mathrm{N}$-cadherin and vimentin. Once EMT occurs, the intercellular adhesion complexes in epithelial cells are disrupted and at the same time, the apico-basal polarity is lost. The basal cytoskeleton disorganizes and the basement membrane is broken down by proteases. EMT induces cells to achieve migratory and invasive properties which allow them to migrate through the extracellular matrix (7). TGF $\beta /$ Smads, Wnt/ $\beta$-catenin and Notch pathways induce EMT by targeting transcription factors including Snail, Slug, Zeb1/2 and Twist (8). In addition, receptor tyrosine kinases (RTKs), including fibroblast growth factor (FGF) and epidermal growth factor (EGF), can also promote the migration and invasion of cancer cells and induce EMT in malignancies.

FOXO1 is one of the members of the FOXO subfamily of forkhead transcription factors (9) and participates in various cellular events such as cell cycle and apoptosis control (10). As a tumor-suppressor, FOXO1 expression is low in endometrioid endometrial cancer cells (11) and non-small cell lung cancer (12) and inhibits prostate cancer cell migration and invasion (13). Recently, FOXO1 was proven to play key roles in drug resistance in tongue (14), non-small cell lung (15), ovarian (16) and prostate cancer (17).

FYN, a 59-kDa protein, is one of the members of the Src family of kinases (SFKs) which are types of non-receptor tyrosine kinases (NRTKs) overexpressed in various types of cancers (18). FYN contains four domains including SH1, $\mathrm{SH} 2, \mathrm{SH} 3$ and SH4 (19), which is similar with other members of the SFKs such as Src, Lyn, Lck and Yes (20). The SH1 domain is the C-terminal domain with kinase activity, $\mathrm{SH} 2$ 
is a structurally conserved region taking part in protein interactions, which can bind to phosphotyrosine residues with the pYEEI sequence, while another highly conserved domain SH3 can recognize PXXP-like sequences. The N-terminal domain SH4 is associated with the cell membrane through palmitoylation or myristoylation (21). FYN plays important roles in Alzheimer's disease by regulating $A \beta$ production, mediating $A \beta$-induced synaptic deficits and neurotoxicity and inducing tyrosine phosphorylation of tau. In addition, FYN can regulate $\mathrm{T}$ cell development and $\mathrm{T}$ cell receptor signal transduction. In addition, FYN is overexpressed in prostate cancer (22), and participates in cell growth, migration and apoptosis as a downstream target of the PI3K/AKT pathway (23). Recently, FYN was found to play a key role in the Wnt receptor Frizzled2 which drives EMT (24), and to predict early recurrence in patients treated with endocrine therapy as an important molecule in tamoxifen resistance (25). However, the role and mechanism of FYN in breast cancer progression are still unclear.

In the present study, we showed that FYN was overexpressed in breast cancer and promoted breast cancer cell proliferation, migration and invasion. Furthermore, FYN was transcriptionally inhibited by FOXO1 and mediated FGF2-induced EMT through both the PI3K/AKT and ERK/MAPK pathways.

\section{Materials and methods}

Cell culture. MCF10A, MDA-MB-231, MDA-MB-435, MCF-7 and T47D cell lines were purchased from the Cell Bank of the Type Culture Collection of the Chinese Academy of Sciences (Shanghai, China). MDA-MB-231 and MDA-MB-435 cell lines were cultured in RPMI-1640 medium with $10 \%$ fetal bovine serum (FBS) (both from Gibco, Grand Island, NY, USA) while MCF-7 and T47D cell lines were supplemented with Dulbecco's modified Eagle's medium (DMEM) (HyClone, Logan, UT, USA) with $10 \%$ FBS. MCF10A cells were maintained in DMEM/F12 medium (HyClone) supplemented with $5 \%$ horse serum (Gibco), $10 \mathrm{mg} / \mathrm{ml}$ insulin, $0.5 \mathrm{mg} / \mathrm{ml}$ hydrocortisone (both from Sigma-Aldrich, St. Louis, MO, USA), 20 ng/ml EGF (R\&D Systems, Minneapolis, MN, USA), and $100 \mathrm{ng} / \mathrm{ml}$ cholera toxin (Sigma-Aldrich). All of the cell lines were supplemented with $1 \%$ penicillin/streptomycin (Gibco), in a $5 \% \mathrm{CO}_{2}$ and humidified atmosphere at $37^{\circ} \mathrm{C}$.

Antibodies, reagents and transfection. The anti-ERK1/2, anti-phospho-ERK1/2, anti-AKT, anti-phospho-AKT, anti-FAK, anti-phospho-FAK and anti-Snail antibodies were purchased from Cell Signaling Technology (Beverly,MA, USA). The anti-E-cadherin, anti-cytokeratin 19, anti-N-cadherin, anti-Slug, anti-FYN, anti-vimentin and anti-nestin antibodies were purchased from Abcam (Cambridge, MA, USA). The siRNAs targeting FYN or the control were obtained from Santa Cruz Biotechnology (Santa Cruz, CA, USA). FGF2 was purchased from R\&D Systems. PP1, BGJ398, AZD8931, LY294002 and PD98059 were acquired from Selleck Chemicals (Houston, TX, USA).

Cells were seeded into 6-well plates. The cells were then transfected with the siRNA or plasmid using FuGENE HD (Roche, Mannheim, Germany) according to the manufacturer's instructions.
Real-time quantitative PCR (RT-qPCR). The total RNA was extracted with RNA Plus (Takara Bio, Inc., Otsu, Shiga, Japan) according to the manufacturer's recommendations. Then, the cDNA was synthesized from RNA by PrimerScript RT Reagent kit (Takara) according to the manufacturer's protocol. RT-qPCR was carried out using a SYBR-Green PCR mix (Takara) on a Bio-Rad CFX96 Real-Time PCR system. Quantification of the target gene expression was calculated by normalizing the averaged $\mathrm{Ct}$ value of the target gene to the averaged $\mathrm{Ct}$ value of the housekeeping gene $\beta$-actin $(\Delta \mathrm{Ct})$, and determined as $2^{-\Delta \mathrm{Ct}}$.

Western blotting. Cells were lysed in RIPA lysis buffer containing protease inhibitor cocktail. Protein lysates were resolved by $10 \%$ SDS/PAGE and the separated proteins were transferred onto a polyvinylidene fluoride (PVDF) membrane (Millipore, Bedford, MA, USA). Non-specific binding was blocked by $5 \%$ skimmed milk in Tris-buffered saline and Tween-20 (TBST) for $1 \mathrm{~h}$ at room temperature. The membranes were incubated with the primary antibody at an appropriate dilution overnight at $4{ }^{\circ} \mathrm{C}$ and then incubated with a suitable HRP-conjugated anti-rabbit or anti-mouse secondary antibody at an appropriate dilution at room temperature for $1 \mathrm{~h}$. The blots were visualized with enhanced chemiluminescence (ECL) reagent (Millipore).

Cell proliferation assay. The MTT assay was used to evaluate the ability of cell proliferation. In brief, $5 \times 10^{3}$ cells were seeded into 96-well plates/well. After incubation for the indicated time, the cells were incubated with $10 \mu \mathrm{l}$ MTT $\left(0.5 \mathrm{mg} / \mathrm{ml}\right.$; Sigma-Aldrich) at $37^{\circ} \mathrm{C}$ for $4 \mathrm{~h}$. The medium was then removed, and precipitated formazan was dissolved in $150 \mu \mathrm{l}$ dimethyl sulfoxide (DMSO). The absorbance at $570 \mathrm{~nm}$ was detected using a microplate autoreader.

Transwell assay. The Transwell assay (BD Biosciences, San Diego, CA, USA) was used to evaluate the ability of cell migration and invasion. Briefly, relevant cells were seeded into the top chambers with or without Matrigel (BD). Medium without serum was added to the top chambers, while complete medium was added to the bottom wells. The cells were fixed with $4 \%$ neutral formalin and stained with crystal violet after incubation. The number of migrated cells was counted under a microscope in five predetermined fields.

Immunofluorescence. Cells were seeded onto glass coverslips in 12-well plates. After adherence, the cells were washed three times with phosphate-buffered saline (PBS), then fixed with $4 \%$ neutral formalin and permeabilized with $0.1 \%$ Triton X-100. Non-specific binding was blocked by $3 \%$ BSA in PBS for $1 \mathrm{~h}$ at room temperature and then the membranes were incubated with primary antibody at an appropriate dilution overnight at $4^{\circ} \mathrm{C}$. After being washed with PBS, the membranes were incubated with a suitable FITC-conjugated anti-rabbit or anti-mouse secondary antibody (Cell Signaling Technology) at an appropriate dilution at room temperature for $1 \mathrm{~h}$, then stained with 4',6-diamidino-2-phenylindole (DAPI) (Beyotime, Jiangsu, China) and glass coverslips were observed with a fluorescence microscope. 
Luciferase assay. Cells were transiently transfected with luciferase reporter constructs, mixtures of expression plasmids encoding FOXO1, along with the pRL-TK vector (Promega, Madison, WI, USA). Luciferase activity was measured after $24 \mathrm{~h}$ by the Dual Luciferase Reporter Assay (Promega).

ChIP assay. ChIP assay was performed using kits from Active Motif (Carlsbad, CA, USA). Cells were fixed in formaldehyde. Cells were lysed and nuclei were pelleted by centrifugation. Nuclei were resuspended and sheared using a sonicator (Misonix, Inc., Farmingdale, NY, USA). Sheared chromatin was immunoprecipitated with the anti-FOXO1 or control IgG antibody. The cross-links were reversed overnight at $65^{\circ} \mathrm{C}$ and deproteinated with $20 \mathrm{~g} / \mathrm{ml}$ proteinase $\mathrm{K}$. The DNA samples were semi-quantified by PCR using the following primer sets containing the FOXO1 binding region on the FYN promoter: forward, 5'-CCTTGTTTACTTC-3' and reverse, 5'-TGTTGTT TACNTT-3'.

Statistical analysis. Data are presented as the mean \pm SEM, and were analyzed by one-way analysis of variance (ANOVA). All experiments were carried out at least three times independently to confirm the conclusions. Statistical significance was set at $\mathrm{P}<0.05$.

\section{Results}

FYN is overexpressed in breast cancer and promotes cell proliferation, migration and invasion. Previous studies indicate that FYN is overexpressed in prostate cancer (22), and induces proliferation and migration in the HEK 293T cell line (26). To investigate the role of FYN in breast cancer, we determined the expression of FYN in breast cancer cell lines and the normal breast epithelial MCF10A cells by western blotting (Fig. 1A). The results showed that FYN expression was higher in the MDA-MB-231 and MDA-MB-435 cell lines and in contrast, it was lower in the MCF-7 an MCF10A cell lines.

To further investigate the role of FYN in breast cancer development and progression, we overexpressed FYN by transfecting the FYN plasmid into the MCF10A cell line. We found that FYN expression was significantly higher in the FYN-transfected MCF10A cells by western blotting (Fig. 1B). We next examined the migration and invasion abilities of the the FYN-overexpressed and the control MCF10A cells. MTT analysis showed that MCF10A-FYN cells grew much faster than the control cells (Fig. 1C). We also observed that the number of migrated cells was much higher in the FYN-transfected MCF10A cells than that in the control cells (Fig. 1D).

Conversely, to determine the effect of decreased FYN expression on breast cancer progression, we silenced FYN with siRNAs targeting FYN or inhibited FYN with FYN inhibitor PP1 in the MBA-MB-231 cell line. The FYN expression was significantly decreased in the FYN siRNA-transfected MDA-MB-231 cells when compared to the level in the control cells (Fig. 1E). The MTT assay showed that depletion of FYN inhibited cell proliferation in the MDA-MB-231 cells (Fig. 1F). The Transwell assay showed that depletion of FYN inhibited breast cancer cell migration and invasion in the MDA-MB-231 cells (Fig. 1G), suggesting that FYN promotes breast cancer cell proliferation, migration and invasion.

FYN induces EMT in the breast cancer cells. As previously described, FYN promotes breast cancer cell migration and invasion. We therefore speculated that FYN may be involved in the process of EMT consequently promoting breast cancer metastasis. To test this contention, we next investigated the role of FYN in breast cancer cell EMT. EMT results in loss of epithelial markers and concomitant acquisition of mesenchymal markers. Our results showed that the mRNA expression of E-cadherin was decreased, whereas the expression of $\mathrm{N}$-cadherin and vimentin was significantly increased in the FYN-transfected MCF10A cells by RT-qPCR (Fig. 2A). In addition, the expression of EMT-related transcription factors Slug and Snail was significantly elevated in the FYN-overexpressed MCF10A cells when compared with that of the control cells (Fig. 2A). Furthermore, overexpression of FYN in the MCF10A cells led to decreased expression of epithelial markers E-cadherin and cytokeratin 19 and increased the expression of mesenchymal markers $\mathrm{N}$-cadherin and vimentin by western blotting (Fig. 2B).

In contrast, the expression of mesenchymal markers vimentin and $\mathrm{N}$-cadherin was downregulated and the expression of epithelial marker E-cadherin was upregulated in the FYN-depleted MDA-MB-231 cells by RT-qPCR (Fig. 2C) and western blotting (Fig. 2D). In addition, the expression of EMT-related transcription factors Slug and Snail was significantly reduced in the FYN-depleted MDA-MB-231 cells compared with that of the control cells by RT-qPCR (Fig. 2C) and western blotting (Fig. 2D). Immunofluorescence staining also revealed that the E-cadherin expression was decreased in the FYN-overexpressing MCF10A cells and increased in the FYN-depleted MDA-MB-231 cells, whereas the vimentin expression was increased in the FYN-overexpressing MCF10A cells and decreased in the FYN-depleted MDA-MB-231 cells (Fig. 2E). Moreover, EGF-mediated remodeling of the cytoskeleton from cortical actin to stress fibers was noted, as determined by phalloidin staining (Fig. 2E).

FYN mediates EGF and FGF2-induced EMT. Previous studies have indicated that FYN is a downstream target of the TGFT $\beta /$ Smad pathway and can be upregulated by TGF $\beta$-1 (27). In addition, the TGF $\beta /$ Smad pathway, the receptor tyrosine kinase pathway can also promote cell proliferation, migration and induce EMT. Thus, EGF and FGF2, and their inhibitors AZD8931 and BGJ398 were used to determine whether FYN is a downstream target of the receptor tyrosine kinase pathway. After treatment with EGF or FGF2 for 2 days, the expression of FYN was increased in the MCF10A cell line, while the FYN expression was decreased after treatment with their inhibitors (AZD8931 and BGJ398) (Fig. 3A). Furthermore, FYN expression was decreased in the MDA-MB-231 cells after treatment with AZD8931 or BJ398 (Fig. 3B). Moreover, the FYN expression was negatively related to the E-cadherin expression in the MCF10A cells (Fig. 3A), whereas it was positively related to $\mathrm{N}$-cadherin expression in the MDA-MB-231 cells (Fig. 3B). To further investigate the role of FYN in FGF2 and EGF-induced EMT, we depleted the FYN expression by FYN siRNA and 
A

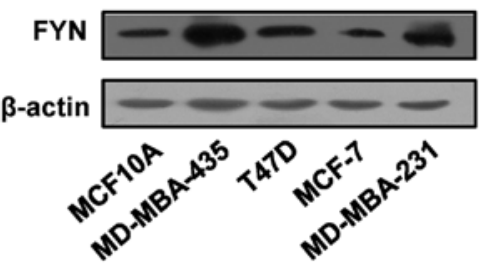

B

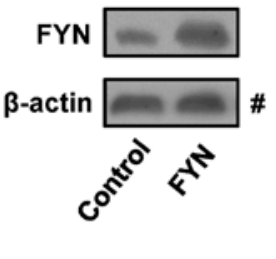

C

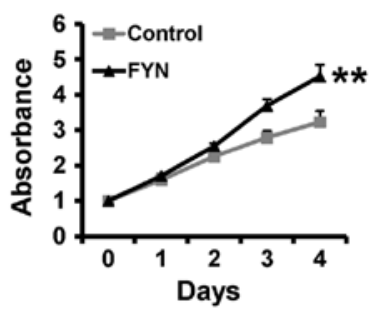

D

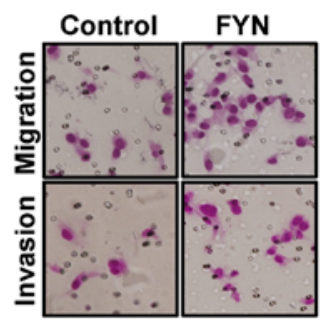

F

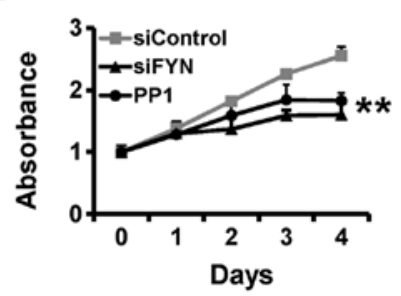

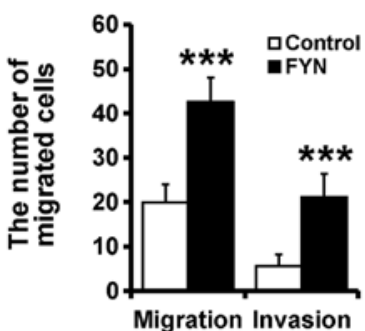

G

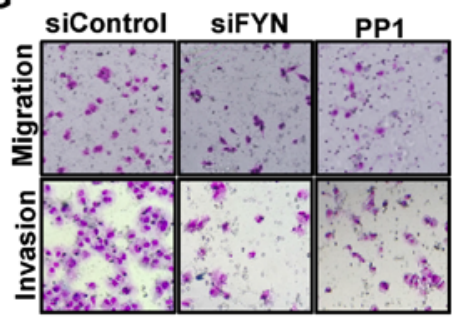

E

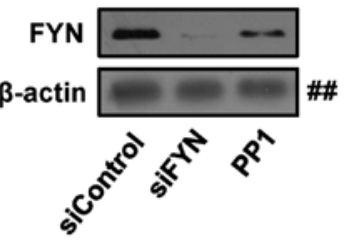

Figure 1. FYN is overexpressed in breast cancer cells and promotes cell proliferation, migration and invasion. (A) FYN expression in breast cancer cell lines as detected by western blotting. (B) Western blotting of FYN expression in FYN-transfected MCF10A and control cells. (C) MTT analysis of FYN-transfected MCF10A and control cells. (D) Transwell analysis of migration and invasion in FYN-transfected MCF10A and control cells. (E) Western blotting of FYN expression in the FYN-depleted MDA-MB-231 and control cells. (F) MTT analysis of FYN-depleted MDA-MB-231 and control cells. (G) Transwell analysis of migration and invasion in the FYN-depleted MDA-MB-231 and control cells.

A

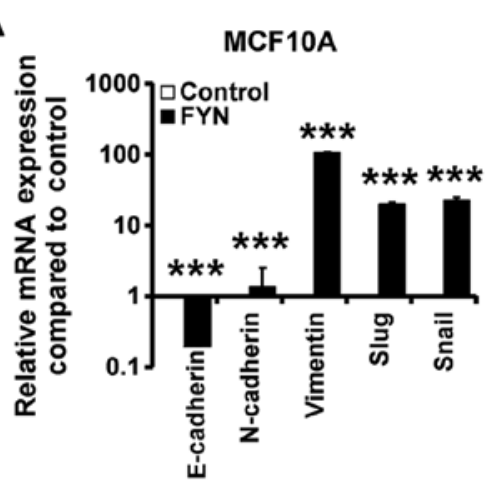

D

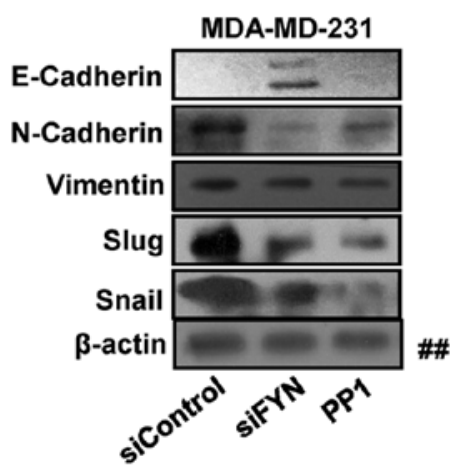

B

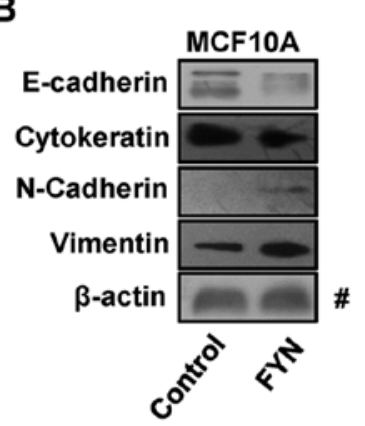

E

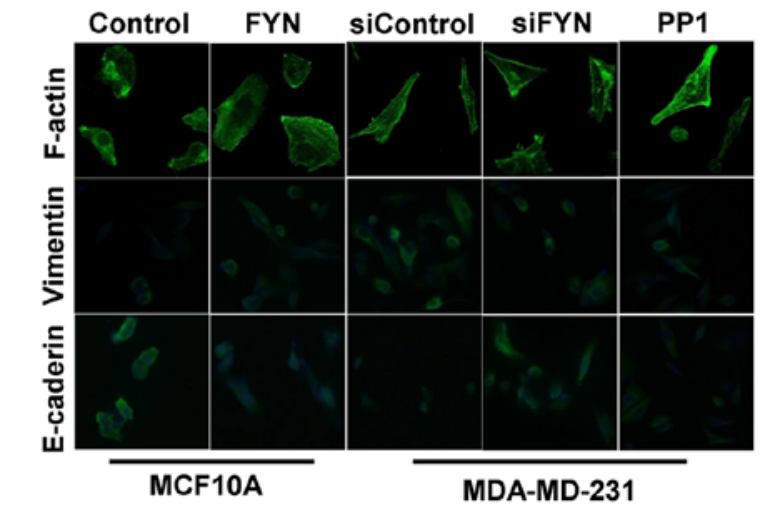

Figure 2. FYN induces EMT in breast cancer cells. (A and C) The expression of epithelial and mesenchymal markers in FYN-transfected MCF10A (A) and FYN-depleted MDA-MB-231 (B) cells by RT-qPCR. (B and D) The expression of epithelial and mesenchymal markers in FYN-transfected MCF10A (C) and FYN-depleted MDA-MB-231 (D) cells by western blotting. (E) Immunofluorescence analysis of vimentin and E-cadherin expression and the morphology and distribution of F-actin in FYN-transfected MCF10A and FYN-depleted MDA-MB-231 cells. 

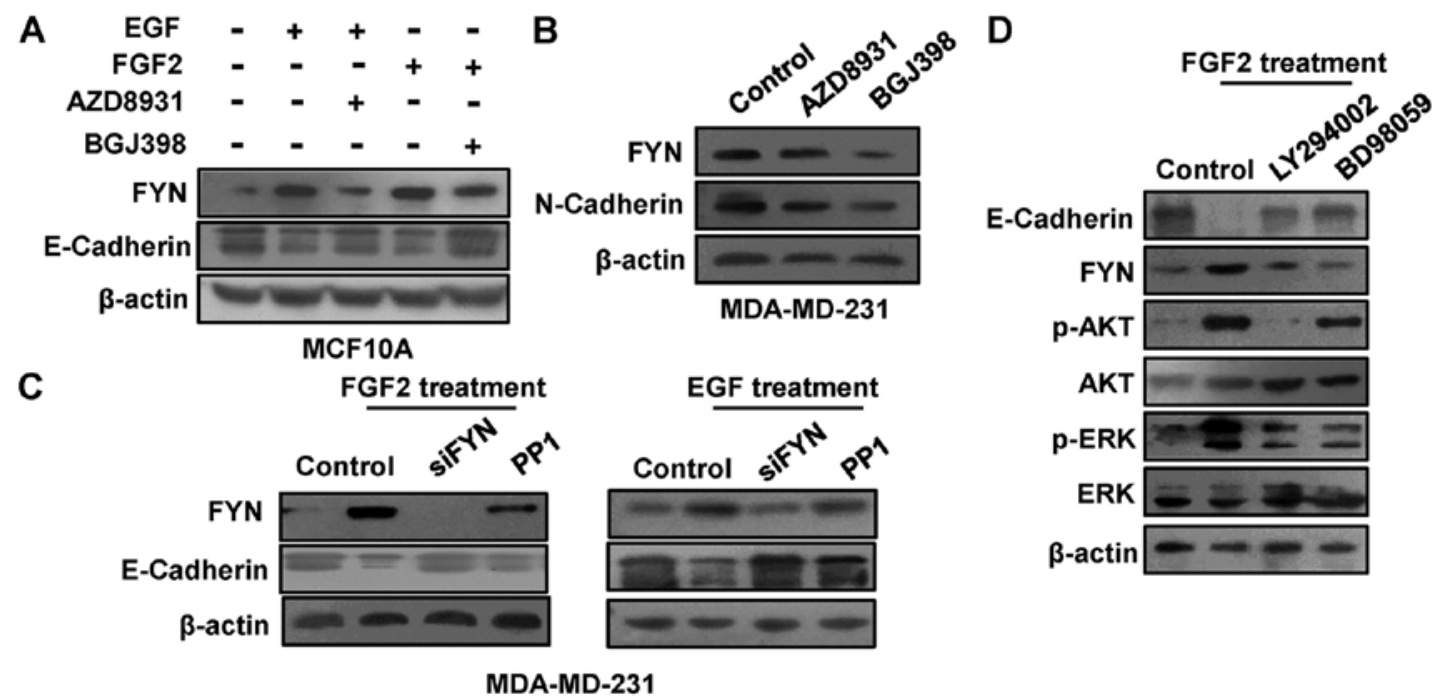

Figure 3. FYN mediates EGF and FGF2-induced epithelial-mesenchymal transition. (A) Western blotting of FYN and E-cadherin expression in the MCF10A cells treated with EGF or FGF2 with or without their inhibitors AZD8931 or BGJ398. (B) Western blotting of FYN and N-cadherin expression in the MDAMB-231 cells treated with AZD8931 or BGJ398. (C) Western blot analysis of FYN and E-cadherin expression in FYN-depleted MDA-MB-231 cells treated with FGF2 (left) or EGF (right). (D) MCF10A cells were incubated with FGF2 and PI3K inhibitor LY294002 or MEK1/2 inhibitor PD98059. The expression of FYN, E-cadherin, and the phosphorylation of AKT and ERK was detected by western blotting.

A

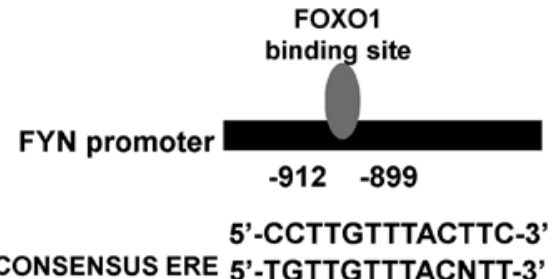

B

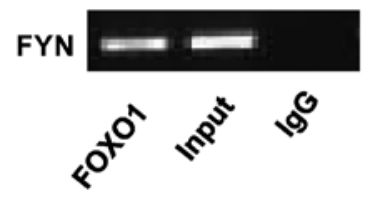

$\mathbf{E}$

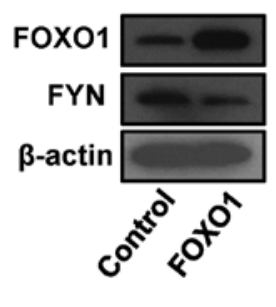

\section{$\mathbf{F}$

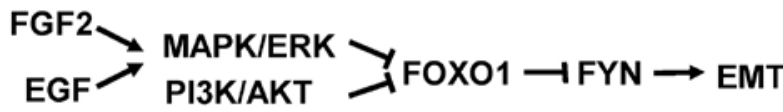

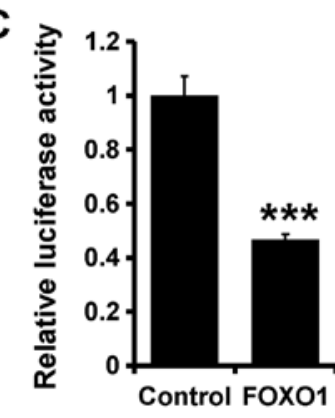

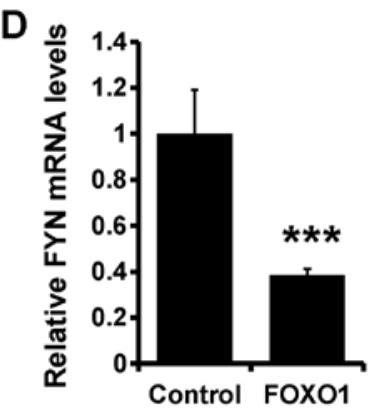

Figure 4. FOXO1 transcriptionally inhibits FYN. (A) FYN promoter with FOXO1 binding site designed for luciferase assay. (B) ChIP assay. Chromatin fragments from MDA-MB-231 cells were immunoprecipitated overnight with the specific anti-FOXO1 antibody or IgG antibody. PCR was performed to detect a putative FOXO1-binding site in the transcriptional regulatory region of FYN. (C) Relative luciferase activity of FOXO1-transfected cells by ChIP assay. (D and E) The expression of FYN in FOXO1-transfected cells by RT-qPCR (D) and western blotting (E). (F) The FYN-related signaling pathway.

inhibitor PP1 after EGF or FGF2 treatment. The results showed that FYN depletion reduced the EGF and FGF2 induced-EMT (Fig. 3C). The PI3K/AKT and ERK/MAPK pathways are involved in FGF2-induced EMT (28). To further investigate the role of FYN in the PI3K/AKT and MAPK/ERK pathways, we examined the FYN expression after treatment with PI3K inhibitor LY294002 or MEK 1/2 inhibitor PD98059. The results showed that FYN expression was decreased after treatment with LY294002 or PD98059 (Fig. 3D).
FOXO1 transcriptionally inhibits FYN. Transcription factor FOXO1 can suppress tumor progression, while PI3K/AKT or ERK/MAPK pathway activation can suppress FOXO1 expression (29). We designed a FYN promoter containing the FOXO1 binding site (Fig. 4A) for luciferase assay to explore the relationship between FYN and FOXO1. ChIP and luciferase assay confirmed that the FOXO1 protein can combine with FYN DNA fragments (Fig. 4B) and inhibit FYN transcription (Fig. 4C). Furthermore, FOXO1 transfection 
decreased the expression of FYN at both the mRNA (Fig. 4D) and protein levels (Fig. 4E). Thus, we can conclude that FGF2 and EGF can decrease FOXO1 expression through the PI3K/AKT and ERK/MAPK pathways, which suppress the transcriptional inhibition of FYN and induce EMT (Fig. 4F).

\section{Discussion}

Previous studies have found that FYN is overexpressed in a variety of solid tumors and hematologic malignancies, such as prostate cancer, squamous cell carcinoma of the head and neck, melanoma and chronic myeloid leukemia $(22,30,31)$. Consistent with these studies, we found that FYN was upregulated in breast cancer cells when compared to the levels in normal breast cells. Moreover, as a member of the SRC family, FYN overexpression in normal cells was capable of changing cell morphology. For instance, overexpression of FYN in normal fibroblast NIH3T3 cells induced anchorage-independent growth and morphological transformation, even in the fully tumorigenic phenotype (32). In the present study, we observed that FYN was highly expressed in the high-invasive breast cancer cell lines (MDA-MB-231 and MDA-MB-435) than in the low-invasive breast cancer cell lines (MCF-7 and T47D) and in the normal breast mammary cell line MCF10A. In addition, it has been reported that FYN plays an important role in integrin-mediated cell adhesion and migration (33). Depletion of FYN reduced cell migration and invasion in 293T cells (26). Consistent with previous studies, we observed that depletion of FYN in the highly invasive MDA-MB-231 cells reduced cell migration and invasion. Furthermore, overexpression of FYN increased cell motility in the low-invasive MCF10A cell line, suggesting that FYN promotes breast cancer cell migration and invasion.

E-cadherin plays a crucial role in epithelial cell-cell adhesion and loss of E-cadherin can be regarded as a hallmark of EMT (34). Interferon- $\gamma$ was found to reduce the expression of EMT epithelial marker E-cadherin in a FYN-dependent manner, which can be inhibited by Src kinase selective inhibitor PP1 (35). TGF- $\beta 1$ was proven to repress E-cadherin expression in A549 cells through the Fyn-p38-Snail signaling pathway (36). In the present study, we also observed that E-cadherin was repressed in FYN-overexpressing MCF10A cells and was elevated in the FYN-depleted MDA-MB-231 cells. Snail and Slug, as EMT-related transcription factors and EMT inducers, were able to repress E-cadherin expression (37). We found that FYN upregulated Snail and Slug expression in the breast cancer cells. In addition to E-cadherin, Slug also induced the expression of an important EMT mesenchymal marker vimentin (38). Our results indicated that overexpression of FYN induces EMT processes in human breast cancer cells, including upregulation of mesenchymal markers ( $\mathrm{N}$-cadherin and vimentin) and downregulation of epithelial markers (E-cadherin and cytokeratin 19). These studies indicated that FYN promotes breast cancer cell proliferation, migration and invasion through induction of EMT.

Previous study have indicated that the PI3K/AKT and ERK/MAPK pathways are regulated by FGF2 (28) and Ras induces the expression of FYN through the PI3K/AKT signaling pathway (23). In the present study, we found that FYN was the downstream target of the receptor tyrosine kinase pathway. After FGF2 stimulation, the expression of FYN and the phosphorylation level of AKT and ERK were increased, while the expression of E-cadherin was decreased. Moreover, the expression of FYN and E-cadherin was decreased after treatment with the PI3K or MEK1/2 inhibitor, suggesting that FGF2 regulates FYN expression through both the PI3K/AKT and ERK/MAPK pathways. In addition, FYN was found to be able to induce the activity of PI3K/AKT (26) and ERK/MAPK (39), which indicates the existence of a crosstalk between PI3K/AKT, ERK/MAPK and FYN.

Tumor suppressor FOXO1 is in the downstream of the PI3K/AKT and MAPK/ERK pathways and it can be activated by PI3K/AKT and MAPK/ERK inhibition (29). We found that FOXO1 decreased the expression of FYN by inhibiting FYN transcription. FOXO1 was previously found to inhibit Runx 2 transcriptional activity and prostate cancer cell migration and invasion (13). In the present study, FOXO1 regulated FGF2-inducing EMT in breast cancer cells by transcriptional inhibition of FYN.

In addition to cell proliferation, migration and invasion, FYN is also involved in apoptosis and drug resistance, however, the mechanisms of these functions of FYN are still unclear, and more studies must be performed to solve these issues. As research progresses, FYN may be regarded as a novel biomarker for cancer prognosis and diagnosis.

\section{Acknowledgements}

The present study was supported by the National Natural Science Foundation of China (nos. 81372843, 81472472 and 81502518), the National Science and Technology Support Program (no. 2015BAI12B15), and the Tianjin Municipal Natural Science Foundation (no. 13JCYBJC21800).

\section{References}

1. Ha R, Chow D and Wynn R: Global trend in breast cancer imaging research 1992-2012: Bibliometric study. AJR Am J Roentgenol 202: 696-697, 2014.

2. Youlden DR, Cramb SM, Yip CH and Baade PD: Incidence and mortality of female breast cancer in the Asia-Pacific region. Cancer Biol Med 11: 101-115, 2014.

3. Shi XJ, Au WW, Wu KS, Chen LX and Lin K: Mortality characteristics and prediction of female breast cancer in China from 1991 to 2011. Asian Pac J Cancer Prev 15: 2785-2791, 2014.

4. Zhang J, Liang Q, Lei Y, Yao M, Li L, Gao X, Feng J, Zhang Y, Gao H, Liu DX, et al: SOX4 induces epithelial-mesenchymal transition and contributes to breast cancer progression. Cancer Res 72: 4597-4608, 2012.

5. Ansieau S: EMT in breast cancer stem cell generation. Cancer Lett 338: 63-68, 2013.

6. Sarkar FH, Li Y, Wang Z and Kong D: Pancreatic cancer stem cells and EMT in drug resistance and metastasis. Minerva Chir 64: 489-500, 2009.

7. Acloque H, Adams MS, Fishwick K, Bronner-Fraser M and Nieto MA: Epithelial-mesenchymal transitions: The importance of changing cell state in development and disease. J Clin Invest 119: 1438-1449, 2009.

8. Thiery JP, Acloque H, Huang RY and Nieto MA: Epithelialmesenchymal transitions in development and disease. Cell 139: 871-890, 2009.

9. Anderson MJ, Viars CS, Czekay S, Cavenee WK and Arden KC: Cloning and characterization of three human forkhead genes that comprise an FKHR-like gene subfamily. Genomics 47: 187-199, 1998.

10. Gross DN, van den Heuvel AP and Birnbaum MJ: The role of FoxO in the regulation of metabolism. Oncogene 27: 2320-2336, 2008. 
11. Goto T, Takano M,Albergaria A,Briese J,Pomeranz KM,Cloke B, Fusi L, Feroze-Zaidi F, Maywald N, Sajin M, et al: Mechanism and functional consequences of loss of FOXO1 expression in endometrioid endometrial cancer cells. Oncogene 27: 9-19, 2008.

12. Maekawa T, Maniwa Y, Doi T, Nishio W, Yoshimura M, Ohbayashi C, Hayashi Y and Okita Y: Expression and localization of FOXO1 in non-small cell lung cancer. Oncol Rep 22: 57-64, 2009.

13. Zhang H, Pan Y, Zheng L, Choe C, Lindgren B, Jensen ED, Westendorf JJ, Cheng L and Huang H: FOXO1 inhibits Runx2 transcriptional activity and prostate cancer cell migration and invasion. Cancer Res 71: 3257-3267, 2011.

14. Zheng G, Jia X, Peng C, Deng Y, Yin J, Zhang Z, Li N, Deng M, Liu X, Liu H, et al: The miR-491-3p/mTORC2/FOXO1 regulatory loop modulates chemo-sensitivity in human tongue cancer. Oncotarget 6: 6931-6943, 2015.

15. Xu ZH, Shun WW, Hang JB, Gao BL and Hu JA: Posttranslational modifications of FOXO1 regulate epidermal growth factor receptor tyrosine kinase inhibitor resistance for non-small cell lung cancer cells. Tumour Biol 36: 5485-5495, 2015.

16. Wang J, Yang H, Li W, Xu H, Yang X and Gan L: Thioredoxin 1 upregulates FOXO1 transcriptional activity in drug resistance in ovarian cancer cells. Biochim Biophys Acta 1852: 395-405, 2015.

17. Duan X, Kong Z, Liu Y, Zeng Z, Li S, Wu W, Ji W, Yang B, Zhao Z and Zeng G: $\beta$-Arrestin 2 contributes to cell viability and proliferation via the down-regulation of FOXO1 in castration-resistant prostate cancer. J Cell Physiol 230: 2371-2381, 2015.

18. Chang YM, Kung HJ and Evans CP: Nonreceptor tyrosine kinases in prostate cancer. Neoplasia 9: 90-100, 2007.

19. Sato I, Obata Y, Kasahara K, Nakayama Y, Fukumoto Y, Yamasaki T, Yokoyama KK, Saito T and Yamaguchi N: Differential trafficking of Src, Lyn, Yes and Fyn is specified by the state of palmitoylation in the SH4 domain. J Cell Sci 122: 965-975, 2009.

20. Szalmás A, Gyöngyösi E, Ferenczi A, László B, Karosi T, Csomor P, Gergely L, Veress G and Kónya J: Activation of Src, Fyn and Yes non-receptor tyrosine kinases in keratinocytes expressing human papillomavirus (HPV) type 16 E7 oncoprotein. Virol J 10: 79, 2013

21. Lu X, Hu X, Song L, An L, Duan M, Chen S and Zhao S: SH2 domain is crucial for function of Fyn in neuronal migration and cortical lamination. BMB Rep 48: 97-102, 2015.

22. Posadas EM, Al-Ahmadie H, Robinson VL, Jagadeeswaran R, Otto K, Kasza KE, Tretiakov M, Siddiqui J, Pienta KJ, Stadler $\mathrm{WM}$, et al: FYN is overexpressed in human prostate cancer. BJU Int 103: 171-177, 2009.

23. Yadav V and Denning MF: Fyn is induced by Ras/PI3K/Akt signaling and is required for enhanced invasion/migration. Mol Carcinog 50: 346-352, 2011.

24. Gujral TS, Chan M, Peshkin L, Sorger PK, Kirschner MW and MacBeath G: A noncanonical Frizzled2 pathway regulates epithelial-mesenchymal transition and metastasis. Cell 159: 844-856, 2014

25. Elias D, Vever H, Laenkholm AV, Gjerstorff MF, Yde CW, Lykkesfeldt AE3 and Ditzel HJ: Gene expression profiling identifies FYN as an important molecule in tamoxifen resistance and a predictor of early recurrence in patients treated with endocrine therapy. Oncogene, 2014.
26. Ninio-Many L, Grossman H, Shomron N, Chuderland D and Shalgi R: microRNA-125a-3p reduces cell proliferation and migration by targeting Fyn. J Cell Sci 126: 2867-2876, 2013.

27. Yu L, Lin Q, Liao H, Feng J, Dong X and Ye J: TGF- $\beta 1$ induces podocyte injury through Smad3-ERK-NF- $\kappa$ B pathway and Fyn-dependent TRPC6 phosphorylation. Cell Physiol Biochem 26: 869-878, 2010.

28. Hardy KM, Yatskievych TA, Konieczka J, Bobbs AS and Antin PB: FGF signalling through RAS/MAPK and PI3K pathways regulates cell movement and gene expression in the chicken primitive streak without affecting E-cadherin expression. BMC Dev Biol 11: 20, 2011.

29. Roy SK, Srivastava RK and Shankar S: Inhibition of PI3K/AKT and MAPK/ERK pathways causes activation of FOXO transcription factor, leading to cell cycle arrest and apoptosis in pancreatic cancer. J Mol Signal 5: 10, 2010.

30. Saito YD, Jensen AR, Salgia R and Posadas EM: Fyn: A novel molecular target in cancer. Cancer 116: 1629-1637, 2010.

31. Singh MM, Howard A, Irwin ME, Gao Y, Lu X, Multani A and Chandra J: Expression and activity of Fyn mediate proliferation and blastic features of chronic myelogenous leukemia. PLoS One 7: e51611, 2012.

32. Kawakami T, Kawakami Y, Aaronson SA and Robbins KC: Acquisition of transforming properties by FYN, a normal SRC-related human gene. Proc Natl Acad Sci USA 85: 3870-3874, 1988.

33. Yeo MG, Oh HJ, Cho HS, Chun JS, Marcantonio EE and Song WK: Phosphorylation of Ser 21 in Fyn regulates its kinase activity, focal adhesion targeting, and is required for cell migration. J Cell Physiol 226: 236-247, 2011.

34. Polyak K and Weinberg RA: Transitions between epithelial and mesenchymal states: Acquisition of malignant and stem cell traits. Nat Rev Cancer 9: 265-273, 2009.

35. Smyth D, Leung G, Fernando M and McKay DM: Reduced surface expression of epithelial E-cadherin evoked by interferon-gamma is Fyn kinase-dependent. PLoS One 7: e38441, 2012.

36. Kim AN, Jeon WK, Lim KH, Lee HY, Kim WJ and Kim BC: Fyn mediates transforming growth factor-betal-induced down-regulation of E-cadherin in human A549 lung cancer cells. Biochem Biophys Res Commun 407: 181-184, 2011.

37. Wakahashi S, Sudo T, Oka N, Ueno S, Yamaguchi S, Fujiwara K, Ohbayashi C and Nishimura R: VAV1 represses E-cadherin expression through the transactivation of Snail and Slug: A potential mechanism for aberrant epithelial to mesenchymal transition in human epithelial ovarian cancer. Transl Res 162 181-190, 2013.

38. Vuoriluoto K, Haugen H, Kiviluoto S, Mpindi JP, Nevo J, Gjerdrum C, Tiron C, Lorens JB and Ivaska J: Vimentin regulates EMT induction by Slug and oncogenic H-Ras and migration by governing Axl expression in breast cancer. Oncogene 30: 1436-1448, 2011.

39. Lee SJ, Jung YH, Oh SY, Yong MS, Ryu JM and Han HJ: Netrin-1 induces MMP-12-dependent E-cadherin degradation via the distinct activation of PKCalpha and FAK/Fyn in promoting mesenchymal stem cells motility. Stem Cells Dev 23: 1870-1882, 2014. 